Mittheilung aus dem pharmazeutisch-chemischen Laboratorium der technischen Hochschule in Braunschweig.

Von H. Beckurts.

\title{
Ueber alkylthiosulfonsaure Salze von organischen Basen.
}

Von J. Troeger und $O$. Linde.

(Vorläufige Mitteilung.)

(Eingegangen den 28. XI. 1899.)

Wie zuerst von Henry $\mathrm{How}^{1}$ ) nachgewiesen ist, lassen sich die thioschwefelsauren Salze organischer Basen darstellen, indem man entweder die Lösungen der Basen mit etwas Weingeist und Schwefelammon an der Luft einige Stunden bis einige Tage stehen lässt, oder Natriumthiosulfat mit den Salzen der organischen Basen umsetzt. Es sind auf diese Weise eine ganze Reihe von gut krystallisierenden Salzen erhalten worden. In der Litteratur finden wir derartige Salze vom Chinin, Cinchonin, Conchinin, Cinchonidin, Paricin, Strychnin, Brucin, Morphin, Codeïn, Thebaïn aufgeführt. Mit Untersuchungen über das Berberin beschäftigt, haben wir auch das thiosulfonsaure Salz des Berberins dargestellt, das auf Zusatz von Natriumthiosulfat zu einer Lösung von salzsaurem Berberin als gelber Niederschlag ausfällt und durch Umkrystallisieren aus Wasser leicht gereinigt werden kann. Da, wie der eine von uns in Gemeinschaft mit R. Otto durch eine Reihe von Untersuchungen gezeigt hat, vollkommene Analogie zwischen den Thiosulfaten einerseits und den alkylthiosulfonsauren Salzen anderseits besteht, so lag es nahe, dass durch Unsetzung der Salze von organischen Basen mit alkylthiosulfonsaurem Salze sich in analoger Weise alkylthiosulfonsaure Salze der organischen Basen bilden würden. Wir haben zu diesem Zwecke Benzol-, p-Toluol-, $\alpha$-Naphtylund $\beta$-Naphtylthiosulfonsaure Salze mit den Salzen von organischen Basen umgesetzt und sind hierbei zu prächtig krystallisierenden, meist sehr schwer löslichen Salzen gelangt. In den meisten Fällen konnten wir durch Umsetzung der Komponenten direkt die Salze in Krystallen rein erhalten, in einigen Fällen wurden sie durch Umkrystallisieren aus Wasser gereinigt. Da das $\beta$-naphtalinthiosulfonsaure Berberin beim Zusammenfügen der Komponenten quantitativ ausfällt, so haben wir auch, indem wir von der Umsetzung des naphtylthiosulfonsauren Salzes mit Jod Gebrauch machten, eine quantitative titrimetrische Be-

1) Chem. Zentralbl. 1855, 93. 
stimmung des Berberins ausgefüht, die, soweit die Versuche vorliegen, recht zufriedenstellende Resultate lieferte. Ob sich diese Eigenschaft auch weiterhin auf andere Alkaloide übertragen lassen wird, vermögen wir noch nicht zu entscheiden, da ausführliche Untersuchungen in dieser Richtung von uns noch nicht ausgeführt sind. Soweit die Untersuchungen bis jetzt vorliegen, ist allerdings anzunehmen, dass nicht in allen Fällen die Bildung der thiosulfonsauren Salze quantitativ verlaufen wird.

p-Toluolthiosulfonsaures Berberin, $\mathrm{C}_{27} \mathrm{H}_{25} \mathrm{NS}_{2} \mathrm{O}_{6}+\mathrm{H}_{2} \mathrm{O}$ $=\mathrm{C}_{20} \mathrm{H}_{17} \mathrm{NO}_{4} \cdot \mathrm{C}_{7} \mathrm{H}_{7} \mathrm{SO}_{2} \mathrm{SH}+\mathrm{H}_{2} \mathrm{O}$. Fügt man zu einer Lösung von Berberinhydrochlorid eine wässrige Lösung von $p$-Toluolthiosulfonsaurem Kalium, so erhält man einen chromgelben Niederschlag, der beim Abfiltrieren ein vollständig farbloses Filtrat liefert, in dem, sofern das thiosulfonsaure Salz im Ueberschuss angewendet wurde, kein Berberin, wohl aber thiosulfonsaures Kalium sich nachweisen lässt. Durch Umkrystallisieren aus viel heissem Wasser wurde das Salz gereinigt. Beim Erkalten der Lösung scheidet sich das Salz in schönen Krystallen ab. Aus wässriger Lösung findet die Abscheidung des Salzes nicht quantitativ statt, ein Beweis, dass eine quantitative Umsetzung der Komponenten nur dann stattfindet, wenn das thiosulfonsaure Salz im Ueberschuss angewendet wird.

Nachstehende Analysen bestätigen die obige Zusammensetzung.

I. $0,5418 \mathrm{~g}$ des lufttrockenen Salzes verloren beim Erhitzen auf $105^{0}$ $0,0182 \mathrm{~g}$ Wasser, entsprechend $3,36 \% \mathrm{H}_{2} \mathrm{O}$.

II. $0,1092 \mathrm{~g}$ der bei $105^{\circ}$ getrockneten Substanz gaben $0,0971 \mathrm{~g} \mathrm{Ba} \mathrm{SO}$, entsprechend $0,01334 \mathrm{~g} \mathrm{~S}=12,21 \% \mathrm{~S}$.

III. $0,1510 \mathrm{~g}$ der bei $105^{\circ}$ getrockneten Substanz gaben $0,3455 \mathrm{~g} \mathrm{CO}$, entsprechend $0,0942 \mathrm{~g} \mathrm{C}=62,38 \% \mathrm{C}$ und $0,0671 \mathrm{~g} \mathrm{H}_{2} \mathrm{O}$, entsprechend $0,0745 \mathrm{~g} \mathrm{H}=4,93 \% \mathrm{H}$.

IV. $0,2660 \mathrm{~g}$ der lufttrockenen Substanz gaben $6,8 \mathrm{ccm}$ feuchten Stickstoff bei $21,5^{\circ}$ und $760 \mathrm{~mm}$ Druck, entsprechend 2,9\% $\mathrm{N}$.

Berechnet auf die Formel

Gefunden:

$\mathrm{C}_{2} \mathrm{H}_{25} \mathrm{~N} \mathrm{~S}_{2} \mathrm{O}_{6}+\mathrm{H}_{2} \mathrm{O}$ :

$$
\begin{aligned}
\mathrm{H}_{2} \mathrm{O} & =3,33 \% \\
\mathrm{~N} & =2,58 \%
\end{aligned}
$$

Berechnet auf die Formel

$$
\begin{gathered}
\mathrm{C}_{87} \mathrm{H}_{25} \mathrm{~N} \mathrm{~S}_{8} \mathrm{O}_{6}: \\
\mathrm{C}_{27}=324=61,95 \% \\
\mathrm{H}_{25}=25=4,78 \% \\
\mathrm{~N}=14=2,68 \% \\
\mathrm{~S}_{2}=64=12,23 \% \\
\mathrm{O}_{6}=96=18,39 \% \\
\hline 523=100,03 \%
\end{gathered}
$$

$$
\begin{aligned}
\mathrm{H}_{2} \mathrm{O} & =3,36 \% \\
\mathrm{~N} & =2,9 \quad
\end{aligned}
$$

Gefunden:

III.

II.

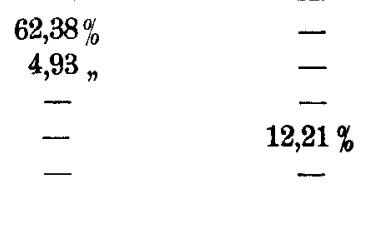


J. Troeger u. O. Linde: Alkylthiosulfonsaure Salze.

B-Naphtalinthiosulfonsaures Berberin, $\mathrm{C}_{30} \mathrm{H}_{25} \mathrm{NS}_{2} \mathrm{O}_{6}=$ $\mathrm{C}_{20} \mathrm{H}_{17} \mathrm{NO}_{4} \cdot \mathrm{C}_{10} \mathrm{H}_{7} \mathrm{SO}_{2} \mathrm{SH}$. Das Salz fällt quantitativ aus, wenn man eine Lösung von überschüssigem naphtalinthiosulfonsauren Salz zu einer Lösung von Berberinhydrochlorid fügt. In Wasser ist das Salz sehr schwer löslich und scheidet sich beim Erkalten der Lösung, allerdings nicht quantitativ, in Form von Krystallnadeln ab.

Nachstehende Analyse bestätigt die obige Zusammensetzung. 0,1578 g Substanz gaben $0,1261 \mathrm{~g} \mathrm{Ba} \mathrm{SO}_{4}$, entsprechend $0,01732 \mathrm{~g} \mathrm{~S}=$ $10,97 \% \mathrm{~S}$.

Die Formel $\mathrm{C}_{80} \mathrm{H}_{25} \mathrm{~N} \mathrm{~S}_{2} \mathrm{O}_{6}$ verlangt:

Gefunden:

$$
11,45 \% \mathrm{~S}
$$$$
10,97 \% \mathrm{~S} \text {. }
$$

Wie wir oben schon erwähnt, haben wir die Umsetzung der thiosulfonsauren Salze mit Jod benutzt, um Berberin titrimetrisch zu bestimmen. Aus der grossen Versuchsreihe mag nur ein Beispiel herausgegriffen werden.

Angewandt wurde eine Lösung, die $1 \mathrm{~g} \beta$-naphtalinthiosulfonsaures Kalium in $300 \mathrm{ccm}$ Wasser gelöst enthielt. Diese Lösung wurde unter Zusatz von etwas Stärkelösung mit 1/100-Normal-Jodlösung titriert. $10 \mathrm{ccm}$ Lösung des thiosulfonsauren Salzes verbrauchten bis zur eintretenden Bläuung $9,95 \mathrm{ccm} \mathrm{1/100-Normal-Jodlösung.} \mathrm{(Die} \mathrm{einzelnen}$ Bestimmungen ergaben sehr übereinstimmende Resultate.) Von dieser Lösung des thiosulfonsauren Salzes, dessen Gehalt durch Titration ermittelt war, wurden $40 \mathrm{ccm} \mathrm{zu} 50 \mathrm{ccm}$ einer Berberinsalzlösung gegeben, die $1 \mathrm{~g}$ salzsaures Berberin in $500 \mathrm{ccm}$ Wasser enthält. Sofort nach Zusammenfügen der Lösungen entsteht ein gelber Niederschlag, der auf dem Filter gesammelt und ausgewaschen wird. Das ablaufende Filtrat ist völlig farblos und klar und enthält das überschüssige angewandte thiosulfonsaure Salz, jedoch kein Berberin. Beim Zurücktïtrieren des Thiosulfates waren 16,85 ccm Jodlösung, entsprechend 17,4 Thiosulfatlösung nötig. Es sind somit gebunden vom Berberin $40-17,4=22,6 \mathrm{ccm}$ der Lösung des Thiosulfates. Die Umsetzung zwischen salzsaurem Berberin und $\beta$-naphtalinthiosulfonsaurem Kalium verläuft aber gemäss nachstehender Gleichung.

$$
\begin{array}{rl}
\left(\mathrm{C}_{20} \mathrm{H}_{17} \mathrm{NO}_{4} \cdot \mathrm{HCl}+4 \mathrm{H}_{2} \mathrm{O}\right) & +\mathrm{C}_{10} \mathrm{H}_{7} \mathrm{SO}_{2} \mathrm{SK}=\mathrm{K} \mathrm{Cl}+4 \mathrm{H}_{2} \mathrm{O} \\
443,5 & 262 \\
+ & \mathrm{C}_{20} \mathrm{H}_{17} \mathrm{NO}_{4} \cdot \mathrm{HS} \cdot \mathrm{SO}_{2} \mathrm{C}_{10} \mathrm{H}_{7} .
\end{array}
$$

Hiernach sind 443,5 $\mathrm{g}$ Berberinhydrochlorid auf $262 \mathrm{~g}$ des Thiosulfates nötig oder $50 \mathrm{ccm}$ der obigen Lösung, die $0,1 \mathrm{~g}$ salzsaures Berberin enthält, erfordert $0,0591 \mathrm{~g} \beta$-naphtalinthiosulfonsaures Salz 
Naphtalinthiosulfonsaures Salz und Jod reagieren aber gemäss der Gleichung.

$$
\begin{aligned}
& 2 \mathrm{C}_{10} \mathrm{H}_{7} \mathrm{SO}_{2} \mathrm{SK} \\
& 2 \times 262 \quad 2 \mathrm{~J}=2 \mathrm{JK}+\left(\mathrm{C}_{10} \mathrm{H}_{7} \mathrm{SO}_{2} \mathrm{~S}\right)_{2} \\
& 2 \times 127 .
\end{aligned}
$$

Es entspricht mithin, wie aus obiger Gleichung leicht abzuleiten, $1 \mathrm{ccm} 1 / 100$-Normal-Jodlösung $=0,00262 \mathrm{~g} \beta$-naphtalinthiosulfonsaurem Kalium. Verbraucht sind zur Berberintällung 22,6 ccm der obigen Thiosulfatlösung, entsprechend $22,49 \mathrm{ccm} \mathrm{1/100-Normal-Jodlösung.} \mathrm{Es}$ sind infolge dessen $22,49 \times 0,00262=\mathbf{0 , 0 5 8 9 5} \mathrm{g} \beta$-naphtalinthiosulfonsaures Kalium zur Fällung des Berberinsalzes verbraucht worden, während aus obiger Gleichung sich 0,0591 g berechnet.

Der vorliegende Versuch liefert ein recht zufriedenstellendes Ergebnis. $O b$ diese titrimetrische Bestimmungsmethode sich auch noch auf anderen Alkaloide übertragen lassen wird, sollen weitere Versuche noch entscheiden.

Benzolthiosulfonsaures Berberin entsteht, wenn man zu einer Lösung von salzsaurem Berberin eine Lösung von benzolthiosulfonsaurem Salz zufügt als eigelber Niederschlag, der aus Wasser, in dem er viel leichter löslich ist als das $p$-toluolthiosulfonsaure Berberin, in feinen Krystallnadeln erhalten wird.

$\alpha$-Naphtalinthiosulfonsaures Berberin. Analog den obigen Salzen dargestellt, bildet es eine gelbe Fällung. Lässt sich aus heissem Wasser, in dem es schwerer löslich als die Benzolverbindung, umkrystallisieren.

p-Toluolthiosulfonsaures Morphin. $\mathrm{C}_{24} \mathrm{H}_{27} \mathrm{NO}_{5} \mathrm{~S}_{2}=\mathrm{C}_{17}$ $\mathrm{H}_{19} \mathrm{NO}_{3} \cdot \mathrm{HS} \cdot \mathrm{SO}_{2} \mathrm{C}_{7} \mathrm{H}_{7}$. Entsteht aus p-Toluolthiosulfonsaurem Kalium und salzsaurem Morphin als weisse Fällung, die beim Umkrystallisieren aus heissem Wasser feste kompakte Krystalle liefert.

Nachstehende Analyse bestätigt die angenommene Formel.

A nalyse. $0,1616 \mathrm{~g}$ der getrockneten Substanz gaben 0,1585 g BaSO entsprechend $0,0221974 \mathrm{~g} \mathrm{~S}=13,70 \% \mathrm{~S}$.

Berechnet auf die Formel

$$
\begin{gathered}
\mathrm{C}_{24} \mathrm{H}_{27} \mathrm{NO}_{6} \mathrm{~S}_{2}: \\
\mathrm{S}=13,53 \%
\end{gathered}
$$

Gefunden:

$$
\mathrm{S}=13,70 \%
$$

Benzolthiosulfonsaures Morphin scheint nur aus konc. Lösungen auszufallen. Die Lösung trübt sich nach einiger Zeit und scheidet ein Oel ab, das beim Stehen zu einer weissen Substanz erhärtet, während die wässrige Flüssigkeit glänzende Krystallnadeln abscheidet.

$\beta$-Naphtalinthiosulfonsaures Morphin. Entsteht als ölige Fällung, die aus heissem Wasser anfangs auch wieder ölig sich ab- 
scheidet, beim längeren Stehen aber ein weisses undurchsichtiges Pulver bildet.

p-Toluolthiosulfonsaures Strychnin, $\mathrm{C}_{28} \mathrm{H}_{30} \mathrm{~N}_{2} \mathrm{O}_{4} \mathrm{~S}_{2}=\mathrm{C}_{21}$ $\mathrm{H}_{22} \mathrm{~N}_{2} \mathrm{O}_{2} \cdot \mathrm{HS} \cdot \mathrm{SO}_{2} \mathrm{C}_{7} \mathrm{H}_{7}$. Aus Wasser umkrystallisiert, bildet dasselbe glänzende Krystallblättchen.

Analyse. $0,1167 \mathrm{~g}$ der getrockneten Substanz gaben $0,1075 \mathrm{~g} \mathrm{Ba} \mathrm{SO}$, entsprechend $0,014764 \mathrm{~g} \mathrm{~S}=12,6 \% \mathrm{~S}$.

Berechnet auf die Formel

Gefunden:

$\mathrm{C}_{28} \mathrm{H}_{80} \mathrm{~N}_{9} \mathrm{O}_{4} \mathrm{~S}_{2}$ :

$\mathrm{S}=12,26 \%$

$$
\mathrm{S}=12,6 \%
$$

Wir haben auch andere Salze von organischen Aminen mit thiosulfonsauren Salzen umgesetzt, so z. B. Guanidinchlorhydrat, Anilinchlorhydrat, salzsaures Phenylhydrazin etc. und haben immer sehr gut krystallisierende Salze erhalten. Wir werden diese Untersuchungen auf weitere Alkaloide sowie organische Amine ausdehnen und bitten daher, uns dieses Arbeitsgebiet für einige Zeit zu überlassen.

Mitteilung aus dem pharmazeutisch-chemischen Laboratorium der techn. Hochschule in Braunschweig.

Von H. Beckurts.

\section{Ueber den Alkaloidgehalt javanischer Granatrinde.}

Vor Kurzem hat E. Ewers ${ }^{1}$ ) eine Methode zur Bestimmung des Alkaloidgehaltes in der Granatrinde ausgearbeitet und an der Hand derselben Untersuchungen über den Gehalt der Rinde an Alkaloiden angestellt. Diese Untersuchungen bezogen sich nur auf Rinden südeuropäischen Ursprungs und ergaben, dass in diesen der Alkaloidgehalt ein ziemlich konstanter ist, $-0,5$ bis $0,7 \%$ beträgt. Wurzel und Stammrinden zeigten keinen wesentlichen Unterschied. Die diesbezüglichen abweichenden Angaben werden dadurch erklärt, dass die höheren Werte, welche sich in der Litteratur vorfinden, sich auf javanische Rinden beziehen, und dass die gefundenen niederen Werte bei der Untersuchung von Rinden von mehr oder weniger hohem Alter erhalten wurden. Es war uns damals nicht möglich, auch javanische Rinden in den Kreis unserer Untersuchungen zu ziehen. Nachdem nun solche kürzlich durch Vermittelung des Herrn Apotheker Rathkamp in Batavia zugegangen sind, hat Herr E. Ewers auch über javanische Rinden Untersuchungen angestellt.

1) Diese Zeitschr. 1899, S. 49. 